ARTIGO

\title{
METODOLOGIA UTILIZADA PARA CALCULAR O BLOCO ÓPTICO DE UM SISTEMA DE TRIAGEM DA ACUIDADE VISUAL UTILIZANDO TELA LCD ${ }^{1}$
}

\author{
Fabrício Carvalho Soares ${ }^{2}$ \\ Marcos Pinotti Barbosa
}

\section{RESUMO}

Este trabalho apresenta a metodologia utilizada para calcular o bloco óptico de um sistema de triagem visual utilizando sistema digital com tela LCD. Inicialmente, apresenta os fundamentos da medida da acuidade visual, em especial o padrão definido por Snellen em 1862, baseado na distância e no ângulo de resolução de um optótipo, sendo, até o momento, um dos principais padrões. Define algumas características das telas LCD, principalmente o tamanho do pixel, que, em conjunto com o padrão de Snellen, é uma das variáveis de entrada para o cálculo do bloco óptico. Além do tamanho do pixel, utilizado para calcular o tamanho do optótipo, também é necessário definir as lentes e a distância entre elas, tendo como resultado a ampliação, o tamanho e a distância da imagem formada. Esta metodologia foi aplicada na produção de três protótipos e no estudo de atualização da tecnologia desenvolvida, sendo empregada para diminuir o tamanho do equipamento ou para alterar a potência das lentes para outras encontradas no mercado.

Palavras-chave: Acuidade visual. Bloco óptico. Snellen. LCD.

\section{INTRODUÇÃO}

Há pelo menos três décadas, existem estudos sobre a saúde de crianças em idade escolar e pré-escolar e a influência de alguns fatores no processo de aprendizagem. Entre os principais aspectos estudados, está a acuidade visual. Os problemas visuais respondem por grande parcela de evasão e repetência escolar, pelo desajuste individual no trabalho e por grandes limitações na qualidade de vida, mesmo quando não se trata, ainda, de cegueira. Também é possível observar que diversas vezes a padronização da triagem visual realizada é precária, ou seja, normalmente é feita sem controle rígido da distância e da iluminação, colocando-se um cartaz com letras em tamanho predefinido a uma distância de seis metros. Além disso, também é desejável que o sistema de triagem visual seja digital, sendo capaz de alimentar um banco de dados durante os exames de triagem (SOARES, 2009).

\footnotetext{
${ }^{1}$ Como citar este artigo: SOARES, Fabrício Carvalho; BARBOSA Marcos Pinotti. Metodologia utilizada para calcular o bloco óptico de um sistema de triagem da acuidade visual utilizando tela LCD. ForScience: revista científica do IFMG, Formiga, v. 6, n. 1, e00148, jan./jun. 2018.
}

${ }^{2}$ Autor para correspondência: Fabrício Carvalho Soares, IFMG, e-mail: fabricio.soares@ifmg.edu.br. 
Este trabalho teve como objetivo apresentar a metodologia utilizada para calcular um bloco óptico de um sistema de triagem da acuidade visual, utilizando como fonte de imagem uma tela LCD conectada a um sistema de controle, em especial o sistema desenvolvido por Soares e colaboradores (2010) em parceria com o Laboratório de Pesquisa Aplicada a Neurovisão (Lapan), conforme apresentado na Figura 1. Este equipamento é formado por uma carcaça (itens 3, 4 e 5 da FIG. 1a), uma tela LCD (item 1 da FIG. 1a), um sistema de controle (item 2 da FIG. 1a) e pelo bloco óptico (item 3 da Figura 1a). O bloco óptico (FIG. 1b) é o componente responsável por criar uma imagem virtual a uma distância de 6 metros, ou seja, por criar a impressão de que um objeto próximo está a uma distância predefinida para a medição da acuidade visual, devendo, para este equipamento, aumentar o tamanho da imagem formada. Ele é formado por um espaçador rígido (item 16 da FIG. 1b), sistemas de fixação da lente e do bloco óptico (itens 14, 15, 17 e 18 da FIG. 1b), carcaça (item 11 da FIG. 1b) e por dois pares de lentes (itens 12 e 13 da FIG. 1b). Um dos pares utiliza lentes com potência focal positiva, cuja função é ampliar o tamanho da imagem, e o segundo usa lentes com potência focal negativa, que têm como função aumentar a distância virtual entre a imagem e o observador.

O cálculo do bloco óptico deverá apresentar como resultado a potência de cada lente utilizada, as distâncias entre o primeiro e o segundo par de lentes e entre o segundo par de lentes e a tela LCD. Estes dados possibilitam a definição do tamanho do espaçador do bloco óptico e da carcaça do equipamento.

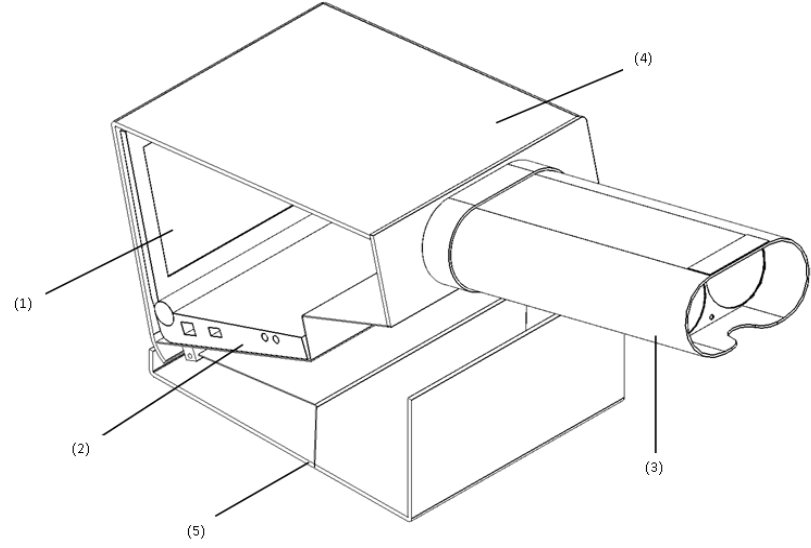

(a)

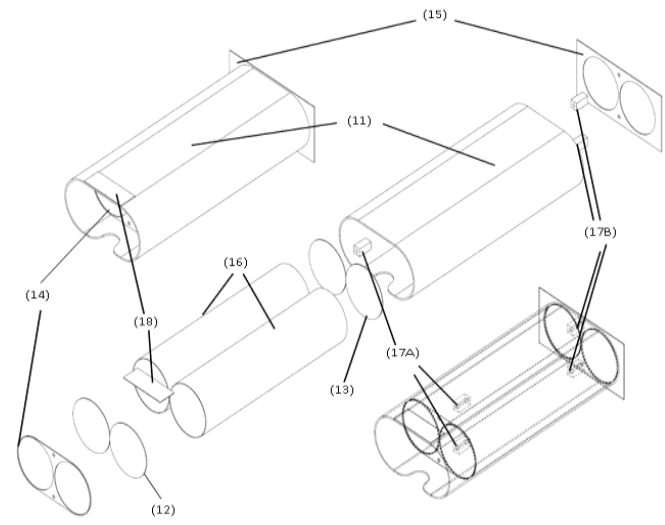

(b)

Figura 1 - Em (a) temos o equipamento desenvolvido em parceria com o Lapan, com destaque para a tela LCD (1) e o bloco óptico (3); em (b) o detalhamento do bloco óptico, onde se destacam os dois pares de lentes (12) e (13).

Fonte: Do autor e colaboradores (2010). 


\subsection{Acuidade Visual: Princípios Básicos}

Acuidade visual é uma característica do olho de reconhecer dois pontos próximos. Sua medida é dada pela relação entre o menor objeto (optótipo) visualizado e a distância entre observador e objeto. É definida como a habilidade de ler uma série de optótipos-padrões que podem ser reconhecidos a uma certa distância, e, normalmente, é uma medida relativa à visão normal (EVANS, 2006).

O limiar de percepção visual é influenciado pela diferença de intensidade de iluminação entre o objeto e o fundo (contraste), a frequência espacial (inverso da espessura da linha em optótipos regulares) e a área do objeto. Em condições de alto contraste e alta iluminação, variação moderada no contraste ou na iluminação representará pequena modificação, de maneira insignificante, na acuidade visual (FRIENDLY, 1978).

Testes de acuidade visual, utilizando a leitura, são realizados deste antes da Idade Média. As maiores mudanças começaram a ocorrer no século XIX, sendo que, atualmente, ainda é utilizado o padrão descrito por Snellen (1862):

A distância máxima que cada optótipo é reconhecido $(d)$ dividida pela distância que deveria estar para ter um ângulo de 5 minutos $(D)$ resulta na fórmula da acuidade visual $(V)$.

$$
V=\frac{d}{D}
$$

Se $d$ e $D$ forem iguais e o optótipo XX for visível a 20 pés, então $V=\frac{20}{20}=1$; em outras palavras, esta é a acuidade visual normal. Se, ao contrário, $d$ for menor que $D$ e se o optótipo XX somente é visível a 10 pés, o optótipo X somente a 2 pés, o optótipo VI somente a 1 pé, estes três casos, então, são expressados respectivamente:

$$
\begin{gathered}
V=\frac{10}{20}=\frac{1}{2} \\
V=\frac{2}{10}=\frac{1}{5} \\
V=\frac{1}{6}
\end{gathered}
$$

$d$, em alguns casos, pode ser maior que $D$, e o optótipo XX ser visível em uma distância maior que 20 pés. Nestes casos, a acuidade visual é maior que a média normal (Traduzido de SNELLEN, 1862).

Tendo o optótipo-padrão 5 partes e considerando-se o proposto por Snellen, o ângulo mínimo de resolução (AMR) será de 1 minuto de arco, conforme pode ser observado na FIG. 2 a seguir. 


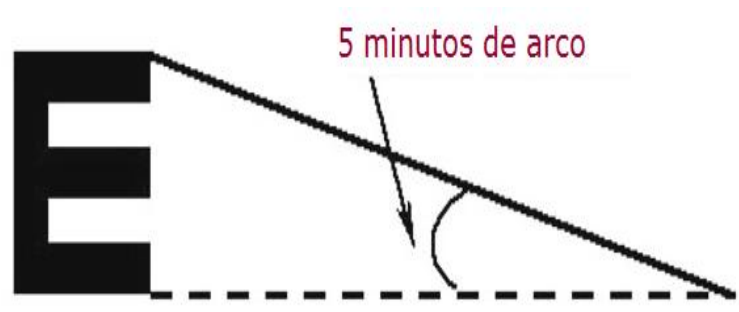

(a)
$\mathrm{AMR}=1$ minuto de arco

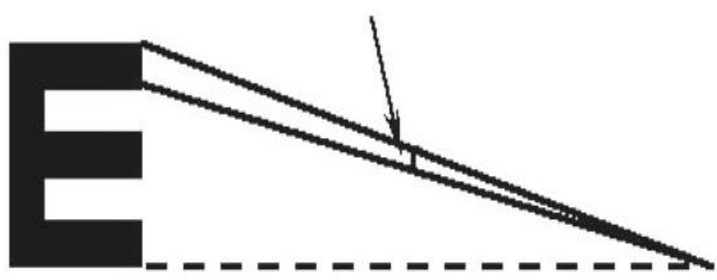

(b)

Figura 2 - Em (a) temos uma letra E com tamanho total de 5 minutos de arco. Em (b) a representação de um segmento da letra contendo 1 minuto de arco.

Fonte: Kolb, et al. $\left(2007^{3}\right)$.

\subsection{Tela LCD: características}

Uma tela LCD é formada por uma matriz de "pontos" quadrados, chamados de pixels, sendo cada pixel formado por três barras: uma vermelha, uma verde e uma azul. A FIG. 3 apresenta uma pequena área de um monitor, contendo 85 pixels de largura $\left(l_{1}\right)$ por 65 pixels de altura $\left(l_{2}\right)$, com uma diagonal aproximada de $3 \mathrm{~cm}(d)$, e, ao lado, um destaque de 20 pixels, mostrando a formação de cada pixel pelas barras vermelha, verde e azul.

As principais características físicas de um monitor LCD são:

a) o tamanho da sua diagonal, normalmente informado em polegadas;

b) a sua resolução, expressa em pixels na horizontal versus pixels na vertical;

c) a relação de aspecto, indicando a relação de tamanho na horizontal pelo tamanho na vertical;

d) o tamanho de cada pixel, em milímetros ou polegadas;

e) a densidade de pixels por milímetro ou polegada;

f) brilho e contraste máximos.

\footnotetext{
${ }^{3}$ webvision.med.utah.edu
} 

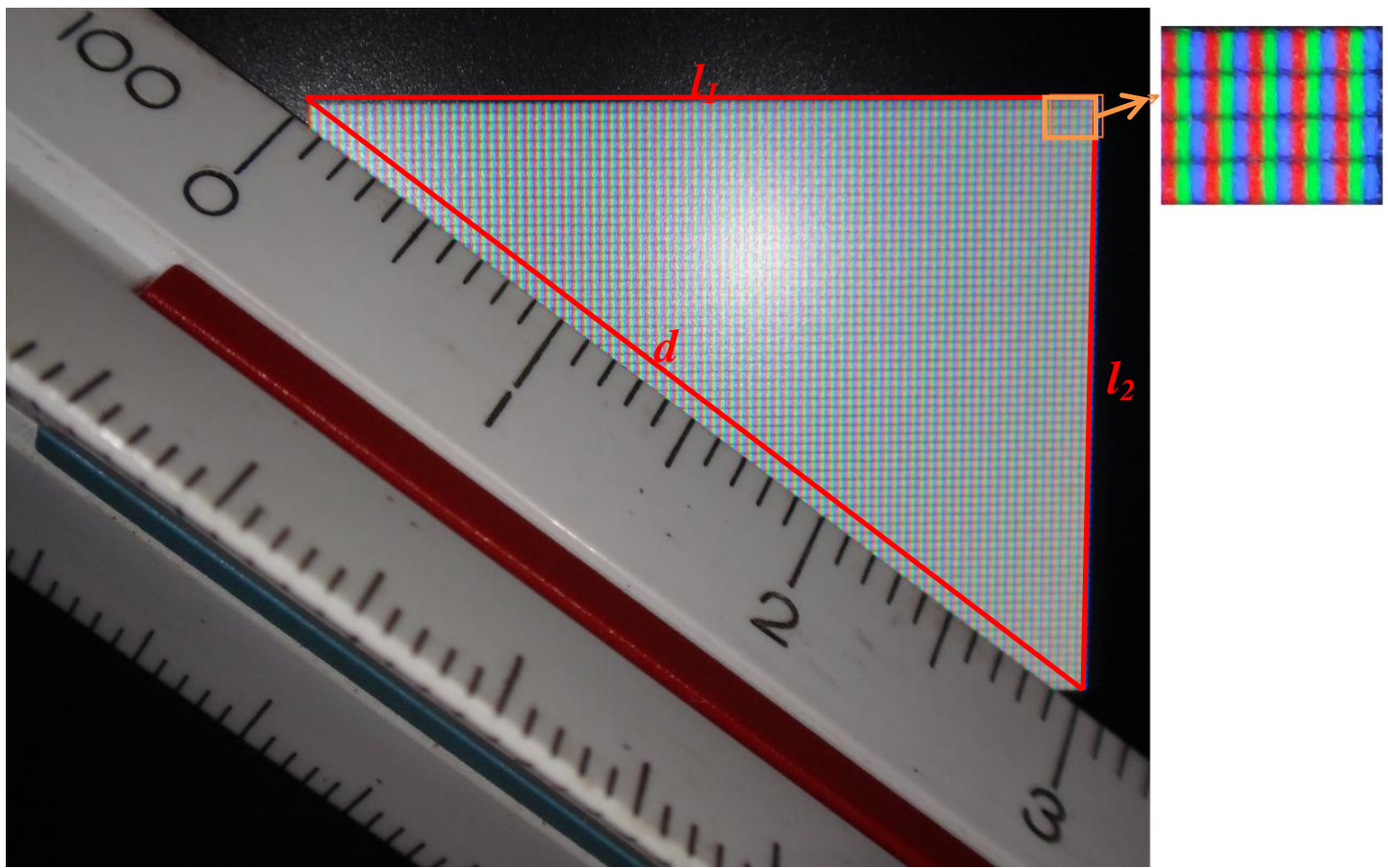

Figura 3 - Área acesa na cor branca de um monitor LG modelo 22LG30R, com diagonal aproximada de $3 \mathrm{~cm}, 85$ pixels de largura e 65 de altura. Destaque no canto superior da imagem mostrando 20 pixels e a sua composição com retângulos nas cores vermelha, verde e azul

\section{METODOLOGIA}

Para calcular o bloco óptico, é necessário definir alguns parâmetros:

a) a distância em que a imagem virtual do optótipo deve estar posicionada será igual a 6,0 m (aproximadamente 20 pés), conforme sugerido por Snellen (1862);

b) o tamanho da imagem virtual (altura do optótipo) para uma letra correspondendo à acuidade visual 1,0 será de $8,72 \mathrm{~mm}$, sendo este valor obtido a partir da distância definida e do ângulo de acuidade visual normal (1' para cada segmento, e 5' para o optótipo), conforme metodologia descrita por Bicas (2002);

c) as lentes utilizadas serão consideradas lentes delgadas, ou seja, terão sua espessura desprezada nos cálculos;

d) o tamanho da letra exibida na tela será de 10 pixels, devendo cada linha do optótipo ter espessura mínima de 2 pixels, minimizando as deformações em formas arredondadas que apareceriam se fosse utilizada a espessura de 1 pixel;

e) o tamanho do pixel da tela LCD: calculado a partir do tamanho e da resolução da tela, conforme metodologia a seguir. 


\subsection{Cálculo do tamanho do pixel de um monitor LCD}

A partir da FIG. 3 e utilizando o Teorema de Pitágoras, é possível calcular o tamanho de cada pixel ( $t p$ ) desta área do monitor, considerando-se um monitor com diagonal medindo $3 \mathrm{~cm}(d)$ e resolução de $85\left(l_{1}\right)$ x $65\left(l_{2}\right)$ pixels, conforme apresentado no Quadro 1:

\begin{tabular}{|c|c|}
\hline Quantidade de pontos na diagonal & Tamanho do pixel \\
\hline$d^{2}=l_{1}^{2}+l_{2}^{2}$ & $t p=\frac{\text { tamanho }}{\text { pixels }}$ \\
$d^{2}=85^{2}+65^{2}$ & $t p=\frac{30 \mathrm{~mm}}{107 \text { pixels }}$ \\
$d=\sqrt[2]{7225+4225}$ & $t p=0,280 \mathrm{~mm} / \mathrm{pixel}$ \\
$d=107$ pixels &
\end{tabular}

Quadro 1 - Cálculo do tamanho de ponto a partir da FIG. 3

Ressalta-se que o valor de pixels não é equivalente à quantidade de pixels cortados pela diagonal da tela, e sim à quantidade que caberia nesta diagonal utilizando-se a medida de um de seus lados. Esta metodologia de cálculo também pode ser utilizada com o tamanho da diagonal de uma tela LCD e a sua resolução, minimizando erros e aproximações de medida. Repetindo a metodologia do Quadro 1 e utilizando as dimensões nominais da tela, cuja diagonal é de 22 polegadas e a resolução é de 1680 x 1050 pixels, o resultado do cálculo do tamanho do pixel foi igual a $0,282 \mathrm{~mm}$, sendo este valor coerente com o encontrado inicialmente.

Para o equipamento de triagem da acuidade visual desenvolvido, foram escolhidos os tamanhos de tela de 8,9 polegadas, com resolução de 1024 x 600 pixels, e de 7 polegadas, com resolução de 800 x 480 pixels e de 1280 x 800 pixels, ambas resoluções disponíveis no mercado, conforme apresentado na TAB. 1.

Tabela 1 - Tamanho do pixel e da letra a ser apresentada na tela (10 pixels) para telas de 8,9 polegadas com resolução de 1024x600 e para telas de 7 polegadas com resolução de 800x480 e 1280x800

\begin{tabular}{lccc}
\hline Tela & Tamanho do pixel $(\mathbf{m m})$ & Tamanho da letra $(\mathbf{m m})$ \\
\hline $8,9 \quad$ polegadas, & resolução & 0,1905 & 1,905 \\
$1024 \times 600$ & & \\
7 polegadas, resolução $800 \times 480$ & 0,1906 & 1,906 \\
7 polegadas, resolução $1280 \times 800$ & 0,1178 & 1,178 \\
\hline
\end{tabular}


Como pode ser observado na Tabela 1, a diferença entre o tamanho do pixel para as telas de 7 polegadas, com resolução de 800x480, e telas de 8,9 polegadas, com resolução de 1024x600, é inferior a $0,1 \%$, sendo consideradas equivalentes para o cálculo do bloco óptico, uma vez que tamanho do pixel é a única grandeza física da tela LCD que influencia neste cálculo.

\subsection{Cálculo do Bloco Óptico}

A partir das definições iniciais, outros parâmetros podem ser calculados. A ampliação (m) que o bloco óptico fornecerá será calculada dividindo-se o tamanho da imagem virtual desejada $\left(h^{\prime}\right)$ pelo tamanho do objeto real $(h)$, conforme a Equação 1 (SMITH, 1997).

$$
m=\frac{h^{r}}{h}
$$

Utilizando-se a Equação 1 para a tela com resolução de 800x480, tem-se que a ampliação $(m)$ do sistema será igual a 4,58.

A partir destas informações e com o auxílio das Equações 2, 3, 4 e 5 (SMITH, 1997), o bloco óptico pode ser calculado.

$$
\begin{aligned}
& u_{j}^{\prime}=u_{j}-y_{j} \times \emptyset_{j} \\
& y_{j+1}=y_{j}+d \times u_{j}^{g} \\
& l_{k}^{\prime}=\frac{-y_{k}}{u_{k}^{g}} \\
& m=\frac{u_{1}}{u_{k}^{g}}
\end{aligned}
$$

Onde: $\phi \quad=$ potência focal da lente (inverso da distância focal);

$d \quad=$ distância entre as lentes;

$j \quad$ = número da lente, para sistema com mais de uma lente;

$k$ = última lente;

$I^{\prime} \quad$ = posição da imagem;

$m$ = ampliação da imagem;

$u \quad=$ ângulo em que o raio chega à lente;

$u^{\prime} \quad$ a ângulo em que o raio parte da lente;

$y \quad=$ altura em que o raio chega à lente. 
Para a definição das lentes, foi estipulado o uso da menor quantidade de lentes possível, independentemente da sua potência. A utilização de um menor número de lentes tem como vantagem facilitar a montagem do equipamento ao minimizar erros de posicionamento e alinhamento. O cálculo do bloco óptico foi feito com o auxílio de uma planilha desenvolvida no LibreOffice Calc (TAB. 2), devendo ser informadas a potência de cada um dos dois pares de lentes e as distâncias entre a tela e o primeiro par de lentes e entre os dois pares de lentes e o tamanho da letra exibida na tela LCD, obtendo como resultados principais a ampliação, a distância e o tamanho da imagem.

Tabela 2 - Fórmulas para cálculo da ampliação, da distância e do tamanho da imagem a partir da potência e das distâncias entre as lentes

\begin{tabular}{|c|c|c|c|c|c|c|}
\hline & A & B & $\mathrm{C}$ & D & $E$ & $\mathrm{~F}$ \\
\hline 1 & & Antes & Lente1 & Meio & Lente 2 & Fim \\
\hline 2 & Potência $(1 / \mathrm{m})$ & & "grau" lente 1 & & "grau" lente 2 & \\
\hline 3 & $\phi(1 / \mathrm{mm})$ & & $\mathrm{C} 2 / 1000$ & & $\mathrm{E} 2 / 1000$ & \\
\hline 4 & d ( $\mathrm{mm})$ & distância lente 1 tela & & Dist. lente 1 lente 2 & & \\
\hline 5 & $y(\mathrm{~mm})$ & & Tamanho letra & & $\mathrm{C} 5+(\mathrm{D} 4 * \mathrm{D} 6)$ & \\
\hline 6 & $\mathbf{u}$ & C5/B4 & & $\mathrm{B} 6-(\mathrm{C} 5 * \mathrm{C} 3)$ & & D6-(E5*E3) \\
\hline 7 & & & & & & \\
\hline 8 & Ampliação & & & & & $\mathrm{B} 6 / \mathrm{F} 6$ \\
\hline 9 & Distância ( $\mathrm{mm})$ & & & & & -E5/F6 \\
\hline 10 & Tamanho (mm) & & & & & F8*C5 \\
\hline
\end{tabular}

Para o cálculo de cada bloco óptico, foram fixados o tamanho da letra e as potências das lentes. As distâncias entre o primeiro par de lentes e a tela e entre os dois pares de lentes foram ajustadas até serem obtidos os valores de tamanho e de distância próximos aos valores de referência para a triagem da acuidade visual. A referência para a medida das alturas $(y)$ do objeto e das imagens é a linha que passa pelo ponto focal das duas lentes. A distância final (linha “9" da TAB. 2) é aquela em que a imagem virtual está formada em relação à Lente 2. O ângulo $(u)$ inicial em que o raio chega à Lente 1 foi definido como a razão entre a altura do objeto (optótipo exibido na tela) e a distância entre o objeto e o primeiro par de lentes (Lente 1), ou seja, que a altura máxima a que o raio chegará à primeira lente será igual à altura do objeto. A amplitude pode ser calculada tanto pela equação [1] quanto pela equação [5], uma vez que o tamanho do objeto e da imagem está relacionado ao ângulo dos raios que partem deles e chegam a um observador (FIG. 2). Foi utilizada a primeira equação de amplitude para a definição dos parâmetros iniciais do cálculo, e a segunda equação, para aferir se os valores encontrados estavam coerentes com o definido inicialmente. 


\section{RESULTADOS}

Fizeram-se simulações com duas telas diferentes, ambas com diagonal de 7 polegadas, sendo a primeira com resolução de 800x480 pixels, e a segunda, 1280x800 pixels.

A TAB. 3 apresenta o resultado para a tela com resolução de $800 \times 480$ pixels, com lentes de potência igual a $-11,5 \mathrm{~m}^{-1} \mathrm{e}+3,0 \mathrm{~m}^{-1}$.

Tabela 3 - Resultados do cálculo utilizando uma tela de 7 polegadas com resolução de 800x480, um par de lentes com potência igual a $-11,5 \mathrm{~m}^{-1}$ e um par de lentes com potência igual a $+3,0 \mathrm{~m}^{-1}$

Antes Lente1 Meio Lente 2 Fim Referência Erro

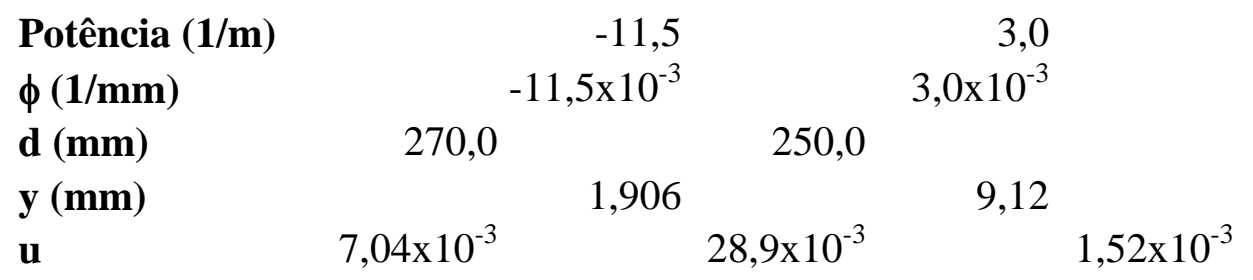

\begin{tabular}{lrrr} 
Ampliação & 4,62 & 4,58 & $0,9 \%$ \\
Distância (mm) & -5994 & -6000 & $0,1 \%$ \\
Tamanho (mm) & 8,81 & 8,72 & $1,0 \%$ \\
\hline
\end{tabular}

Fonte: Do autor (2009).

Conforme pode ser observado na TAB. 3, os valores de distância e tamanho apresentaram erro máximo próximo a $1 \%$ em relação aos valores de referência definidos para a triagem da acuidade visual. O bloco óptico formou uma imagem virtual a $5994 \mathrm{~mm}$ de distância, com altura igual a 8,81 mm. O tamanho mínimo do equipamento é de $520 \mathrm{~mm}$ de comprimento, sendo este valor a soma das distâncias entre a tela e a lente 1 e entre os dois pares de lentes.

Foram produzidos três protótipos (FIG. 4) com as lentes, tamanho de ponto e distâncias, como exibido na Tabela 3, apresentando formação de imagem conforme esperado para a triagem da acuidade visual. Nos dois primeiros protótipos (FIG. 4, itens a e b), estava embarcado na carcaça um computador da marca Asus, modelo EEE PC900, sendo utilizada a sua própria tela, com diagonal de 8,9 polegadas e resolução de 1024 x 600 pixels, tendo como diferença somente as carcaças interna e externa. No terceiro protótipo (FIG. 4, item c), foi utilizada uma tela LCD fabricada pela Innolux, modelo AT070TN92, com diagonal de 7 polegadas e resolução de 800 x 480 pixels. Empregou-se o mesmo sistema óptico nos três 
protótipos, incluindo tanto as lentes quanto as distâncias calculadas, uma vez que as telas utilizadas possuíam tamanho de ponto semelhante, com dimensão aproximada de $0,19 \mathrm{~mm}$.

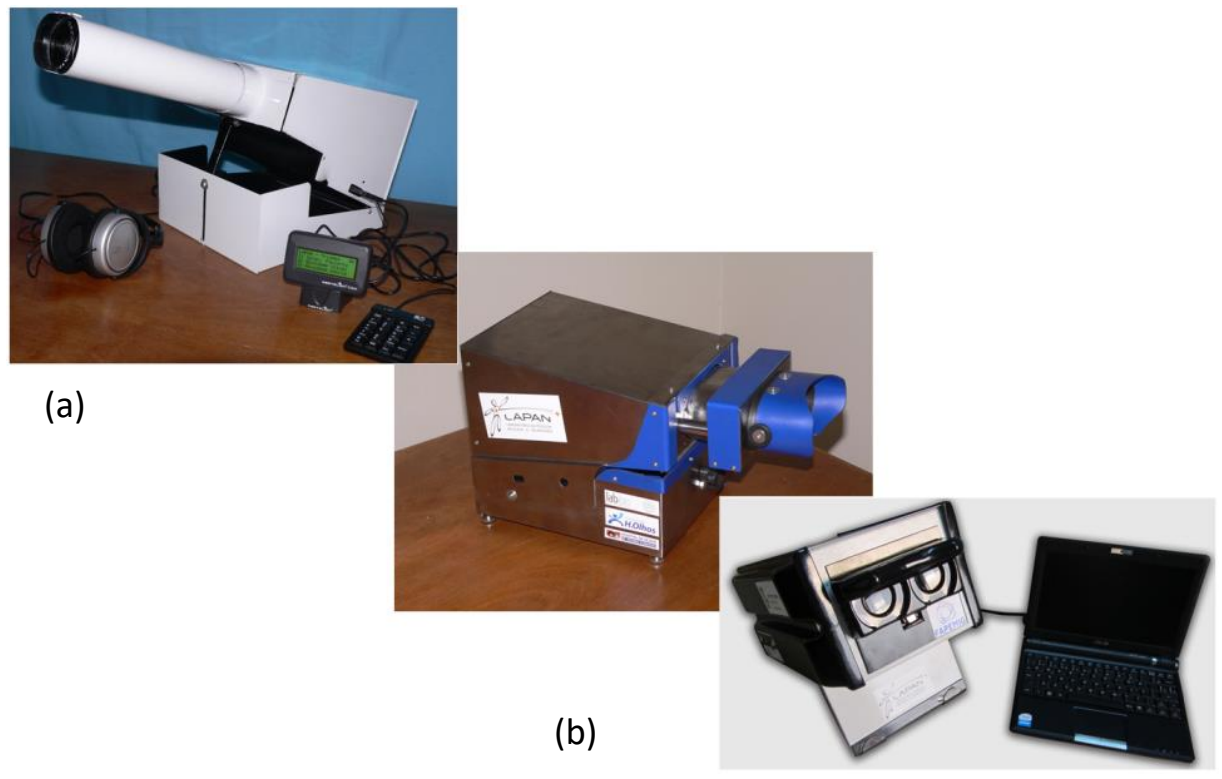

(c)

Figura 4 - Três protótipos produzidos em parceria com o Lapan e a Íon Industria Ltda. Em (a) e (b), dois protótipos com um computador embarcado e em (c) um protótipo com uma tela interna e o sistema de controle externo

Fonte: Do autor.

Com o desenvolvimento de telas LCD, atualmente estão disponíveis no mercado telas com diagonal de 7 polegadas e resolução de 1280x800, apresentando pixels menores em relação às telas utilizadas nos três protótipos. Foram feitos cálculos para a tela de resolução 1280x800 com dois conjuntos de lentes diferentes, conforme apresentado nas TAB. 4 e 5.

Tabela 4 - Resultados do cálculo utilizando uma tela de 7 polegadas com resolução de 1280x800, um par de lentes com potência igual a $-12,0 \mathrm{~m}^{-1}$ e um par de lentes com potência igual a $+4,5 \mathrm{~m}^{-1}$

Antes

Lente

Meio

Lente 2

Fim

Referência Erro

Potência (1/m)

$\phi(1 / \mathbf{m m})$

d (mm)

$\mathbf{y}(\mathbf{m m})$

$\mathbf{u}$

Ampliação

Distância (mm)

Tamanho (mm)
$-12,0$

$-12,0 \times 10^{-3}$

232,0

$5,08 \times 10^{-3}$

1,178
4,50
$4,50 \times 10^{-3}$

153,0

4,12

$$
0,68 \times 10^{-3}
$$

\begin{tabular}{rrr}
7,42 & 7,40 & $0,3 \%$ \\
-6020 & -6000 & $0,3 \%$ \\
8,74 & 8,72 & $0,2 \%$ \\
\hline
\end{tabular}


Tabela 5 - Resultados do cálculo utilizando uma tela de 7 polegadas com resolução de 1280x800, um par de lentes com potência igual a $-5,0 \mathrm{~m}^{-1}$ e um par de lentes com potência igual a $+3,0 \mathrm{~m}^{-1}$

\begin{tabular}{|c|c|c|c|c|c|c|c|}
\hline & Antes & Lente1 & Meio & Lente 2 & Fim & Referência & Erro \\
\hline Potência (1/m) & & $-5,00$ & & 3,00 & & & \\
\hline$\phi(1 / \mathbf{m m})$ & & $-5,00 \times 10^{-3}$ & & $3,00 \times 10^{-3}$ & & & \\
\hline d (mm) & 305,0 & & 195,0 & & & & \\
\hline $\mathbf{y}(\mathbf{m m})$ & & 1,178 & & 3,08 & & & \\
\hline $\mathbf{u}$ & $3,86 \times 10^{-3}$ & & $9,75 \times 10^{-3}$ & & $0,51 \times 10^{-3}$ & & \\
\hline Ampliação & & & & & 7,53 & 7,40 & $1,8 \%$ \\
\hline Distância (mm) & & & & & -6001 & -6000 & $0,0 \%$ \\
\hline Tamanho (mm) & & & & & 8,87 & 8,72 & $1,7 \%$ \\
\hline
\end{tabular}

Ao se utilizar uma tela com pixels menores e manter a potência das lentes próxima à adotada nos protótipos, buscou-se reduzir o tamanho final do equipamento, conforme pode ser visto na TAB.4, tendo como limite mínimo o valor de $385 \mathrm{~mm}$ de comprimento (distância entre a tela e o segundo par de lentes), reduzindo em $135 \mathrm{~mm}$ o tamanho do equipamento em relação aos protótipos. Na TAB. 5, foram fixadas as potências das lentes para valores encontrados no mercado, ou seja, lentes com potência de até $\pm 5 \mathrm{~m}^{-1}$, sem a necessidade de sua fabricação em laboratório especializado, o que resulta em melhor padronização e na redução do custo final do equipamento, uma vez que lentes-padrões custam menos de $20 \%$ do valor das que são fabricadas sob medida. Lentes de menor potência também facilitam a montagem do equipamento, pois são menos suscetíveis a erros de montagem devido a variações nas distâncias entre as lentes e a tela. O tamanho do equipamento, utilizando as lentes apresentadas na TAB. 5, é próximo ao dos protótipos produzidos, com uma redução de apenas $20 \mathrm{~mm}$.

\section{CONCLUSÃO}

A metodologia utilizada para calcular o bloco óptico para um sistema de triagem da acuidade visual é capaz de determinar as características físicas do equipamento, sendo útil, também, como uma ferramenta para estudos de melhoria deste tipo de equipamento.

Esta metodologia foi empregada durante o desenvolvimento de três protótipos utilizando dois tipos diferentes de telas. Também foi usada como ferramenta para atualização da tecnologia desenvolvida utilizando telas de maior resolução, tendo como objetivo 
equipamentos menores, no caso do uso de lentes de alta dioptria, ou com custo de produção otimizado, com as lentes-padrões do mercado.

Os valores apresentados durante este artigo referem-se aos cálculos realizados, podendo haver pequenas diferenças nos protótipos, devido à imprecisão na montagem e na produção das lentes e a pequenas variações do tamanho dos pontos das telas em relação a cada fabricante.

\title{
METHODOLOGY TO CALCULATE THE OPTICAL UNIT OF A VISUAL ACUITY SCREENING SYSTEM BY USING A LCD SCREEN
}

\begin{abstract}
This paper describes the methodology used to calculate the optical unit of a visual acuity screening system by using a LCD screen. It begins with the fundamentals of visual acuity, especially the standard defined by Snellen in 1862, based on distance and angle resolution of an optotype, and, so far, one of the main standards. The fundamentals of visual acuity and some characteristics of LCD screen are presented. Beyond the pixel size used to calculate the size of the optotype, it is also necessary to define the lenses and the distance between them, resulting in magnification, size and distance of the formed image. Three prototypes were produced using this methodology. Furthermore, it has been used to decrease the size of the equipment and/or to change the power of the lenses to others found in the market.
\end{abstract}

Keywords: Visual Acuity. Optical Layout. Snellen. LCD.

\section{REFERÊNCIAS}

BICAS, H. E. A. Acuidade visual: medidas e notações. Arquivos Brasileiros de Oftalmologia, v. 65, p. $375-384,2002$.

EVANS, J. M. Standards for visual acuity. Gaithersburg (USA): NIST, 2006.

FRIENDLY, D. S. Preschool visual acuity screening tests. Transactions of the American Ophthamological Society, v. 76. 1978.

KOLB, H. et al. (Ed.). Webvision: the organization of the retina and visual system. [Bethesda, Md.]: [National Library of Medicine]: [National Center for Biotechnology Information], 2007. Disponível em: <webvision.med.utah.edu>. Acesso em: 15 mar. 2010.

SMITH, W. J. Pratical optical system layout and use of stock lenses. New York: McGrawHill Companies, 1997.

SNELLEN, H. Probebuchstaben zur Bestimmung der Sehschärfe. Utrecht: P.W. van de Weijer, 1862. 
SOARES, F. C. et al. Equipamento binocular digital para a verificação da acuidade visual e do limiar auditivo. BR Pat. 1003231-2, 15 de março de 2010, 25 p.

SOARES, F. C. Sistema de triagem visual e auditiva de crianças em idade escolar, conectado a um banco de dados. 2009. 140 f. Tese (Doutorado em Engenharia Mecânica) Universidade Federal de Minas Gerais, Escola de Engenharia, Belo Horizonte, 2009.

\section{DADOS DOS AUTORES}

\section{Fabrício Carvalho Soares}

E-mail: fabricio.soares@ifmg.edu.br

Currículo Lattes: http://lattes.cnpq.br/4737176760716569

Doutor em Engenharia Mecânica pela UFMG (2009). Mestre em Engenharia Mecânica pela UFMG (2005) e graduado em Engenharia Mecânica pela UFMG (2002). Atualmente é professor do ensino básico, técnico e tecnológico do Instituto Federal de Minas Gerais (IFMG) - Campus Congonhas.

\section{Marcos Pinotti Barbosa}

E-mail: office.pinotti@gmail.com

Currículo Lattes: http://lattes.cnpq.br/1221789114690533

Doutor em Engenharia Mecânica pela Universidade Estadual de Campinas (1996). Mestre em Engenharia Mecânica pela Universidade Estadual de Campinas (1992) e graduado em Engenharia mecânica pela Universidade Estadual de Campinas (1989). Atualmente é professor titular da Universidade Federal de Minas Gerais (UFMG), presidente da Sociedade Latino Americana de Biomateriais, Órgãos Artificiais e Engenharia de Tecidos (SLABO), secretário da Associação Brasileira de Engenharia e Ciências Mecânicas (ABCM) e membro do Conselho de Administração do Parque Tecnológico de Belo Horizonte (BHTEC) como representante do Reitor da UFMG. 